\title{
Energy-saving optimization method for point-to-point trajectories planned via standard primitives in 1-DoF mechatronic systems
}

\author{
Giovanni Carabin ${ }^{1}$ (1) $\cdot$ Renato Vidoni $^{1}$ (1) \\ Received: 5 August 2020 / Accepted: 11 May 2021 / Published online: 17 June 2021 \\ (C) The Author(s) 2021
}

\begin{abstract}
In this work, an analytical methodology to minimize the energy expenditure of mechatronic systems performing point-topoint (PTP) trajectories based on well-known motion primitives is developed and validated. Both PTP trajectory profiles commonly used in industrial motor drives and more complex ones are investigated. Focusing on generic 1-DoF mechatronic systems moving a constant inertia load (e.g., elevators, cranes, CNC machines, Cartesian axis) and possibly equipped or retrofitted with regenerative devices, the consumed energy formulation is firstly derived. Then, the analytical optimization considering all the selected PTP trajectory profiles is computed and a generic closed-form solution is determined. Finally, numerical and experimental evaluations are done showing the effectiveness of the theoretical results and proposed methodology. In addition, all the different trajectories are compared with respect to energy consumption.
\end{abstract}

Keywords Motion-planning $\cdot$ Trajectory optimization $\cdot$ Energy efficiency $\cdot$ Mechatronics

\section{Introduction}

The minimization of energy consumption in manufacturing and industrial processes is becoming an important objective of an engineering design process [1-3]. Indeed, in the recent years, topics such as environmental issues as well as increase of energetic costs have driven politics and public opinion to take solid and challenging actions toward a more sustainable production [4]. The European Union has committed itself to bind a target of saving $20 \%$ of primary energy consumption (in reference to 2007 consumption) by 2020 [5]. This policy has been then extended and a new binding energy efficiency target of 30\% energy savings until 2030 for the European Union has been subscribed [6].

The more attention on this topic can be demonstrated by the increasing number of published works in this area, as reviewed in [7] where different methods and technologies for enhancing the energy performance of mechatronic systems

Giovanni Carabin

giovanni.carabin@unibz.it

Renato Vidoni

renato.vidoni@unibz.it

1 Faculty of Science and Technology, Free University of Bozen-Bolzano, Bolzano, Italy have been classified in three main categories: hardware, software, and mixed approaches.

The hardware approaches consider the modification of parts of existing systems or the design of new ones to create more energy-efficient mechatronic devices. In particular, the availability of new lighter and strength materials makes possible the design of lighter robotic arms [8-11] and components $[12,13]$. Lightweight structures are as well obtained by reducing the moving masses: actuators are moved next to the robot base and the motion is transmitted through a system of pulleys and timing belts [14, 15]. Hardware approaches include also technologies for increasing the components efficiency [16], and for implementing energy recovery [17, 18] and distribution strategies [19]. These allow for new scenarios of reduced energy consumption and therefore less environmental impact.

The software approach, conversely, carries out the energy consumption reduction by optimizing the motion planning phase as well as scheduling the operations. Machines and robots are often operated to maximize production outputs (i.e., minimization of time); this causes both high energy losses at high velocities and surpluses in deceleration. Therefore, the speed motion profile in a PTP operation can be changed [20-26] as well as the path profile in a multi-point trajectory $[27,28]$ in order to use less energy. In literature, focusing only on PTP motions, several works dealing with the optimization and comparison between 
different PTP trajectory profiles, from the most common in industrial application such as the trapezoidal speed profile [29,30] to more complex ones [20-22], can be found. Richiedei et al. [21] propose an analytical method to improve the energy efficiency of 1-DoF constant inertia systems, by selecting both the best motion law for the application and the optimum cycle time. In [23], the PTP trajectory planning of the excavating process of a large cable shovel has been considered: polynomial curves of different degrees have been numerically optimized to reduce the energy consumption per volume unit of dig material and compared in terms of excavating performance with the conventional S-curve. Liu et al. [26] propose a trajectory planning optimization strategy, based on 43-3-4 degree polynomial interpolation, for Delta 3 robot performing high-speed handling operations. In [24], the kinematic redundancy is exploited as a tool to enhance the energetic performance of a robotic cell, while Ayten et al. [25] consider two PTP trajectory optimization methods for redundant/hyper-redundant manipulators. The first method considers the kinematic and dynamic constraints in a sequential manner to avoid running the inverse dynamics, while in the second one a new concept of virtual link is introduced to replace the redundant links and eliminate the impossible configurations before running the inverse dynamic model.

Finally, the mixed approach considers hardware and software modifications of the mechatronic system working in synergy. This is the case of trajectory optimization in systems equipped with regenerative drives to maximize the energy regenerated and stored in the capacitors [31, 32]. Further methods use compliant elements inserted, in parallel or in series, to the actuators, in combination with a proper trajectory optimization to exploit the free vibration response of the system performing cyclic tasks [33-36]. Scalera et al. in [37] present a detailed review of these methods.

This work focuses on the study of the energy consumption and minimization of a generic 1-DoF mechatronic system moving a constant inertia load (e.g., elevators, cranes, CNC machines, Cartesian axis) possibly equipped or retrofitted with regenerative devices (i.e., mixed approach). As a matter of fact, these kinds of systems cover a wide range of applications in production, packaging, and logistic plants.

Although the motion planning optimization to increase the energy efficiency of such a systems is common in literature, few works (e.g., [21-23]) address the problem from an analytical point of view. Following this approach would represent a clear advantage allowing to obtain the solution in a quick and direct way relying only on the knowledge of few system physical parameters. In particular, this would be important for an implementation of the method in an embedded mechatronic system (e.g., motor servo drive), where the computational power is generally limited. With this idea in mind, in this work, different PTP trajectory profiles have been investigated including both motion primitives commonly used in industrial motor drives (e.g., trapezoidal speed profile and double-S profile) and more complex ones (e.g., cycloidal and polynomial profiles). In particular, the developed approach considers the analytical minimization of the energy for all the considered PTP trajectory profiles, thus deriving closed-form solutions. In addition to other similar works, beyond of considering different motion primitives and optimizing the time required to execute a task, this method analyzes and optimizes also the shape of the motion profile (i.e., non-symmetrical profile with non-fixed acceleration and deceleration times are considered). Moreover, an experimental validation of the method has been carried out.

The paper is organized as follows: in Section 2, the model of the 1-DoF generic mechatronic system is presented; it is then exploited in Section 3 to derive the generic formulation of the energy consumption, independent from the motion profile. Afterward, in Section 4, the mathematical definition of all the considered trajectories is derived and substituted into the energy formulation to obtain the consumed energy for each motion profile as well as the optimum solution that minimizes the energy consumption in closedform (Section 5). After that, in Section 6, the different trajectories are compared and evaluated. Finally, numerical and experimental analysis and validation are reported in Sections 7 and 8.

\section{Mechatronic system model}

The methods presented in this work are meant to be applied on a generic 1-DoF mechatronic system moving a constant inertia load. In Fig. 1, an example of a possible application, e.g., a Cartesian robot axis, is shown: an electric motor (e.g., DC motor or permanent magnet synchronous motor - PMSM) moves a payload on an inclined plane (by an angle $\vartheta$ ) over a distance $L$, by means of a toothed belt with a reduction ratio equal to $\tau$. The model takes into account the load inertia, the system Coulomb, and viscous frictions, as well as the resistive losses in the motor windings. Motor iron losses and stray losses are not directly taken into account by the model. Anyway, as stated in [21], they can be fictitiously included by increasing the viscous friction and the motor resistance terms, respectively. Whereas, motor inductance here is not considered since in a point-to-point motion, no dissipation occurs through it [21].

This test-case has been chosen for the derivation and validation of the developed methods. However, there is no loss of generality as methods can be easily adapted to any 1-DoF mechatronic systems with a constant inertia load. 
Fig. 1 Model of the mechatronic system under evaluation

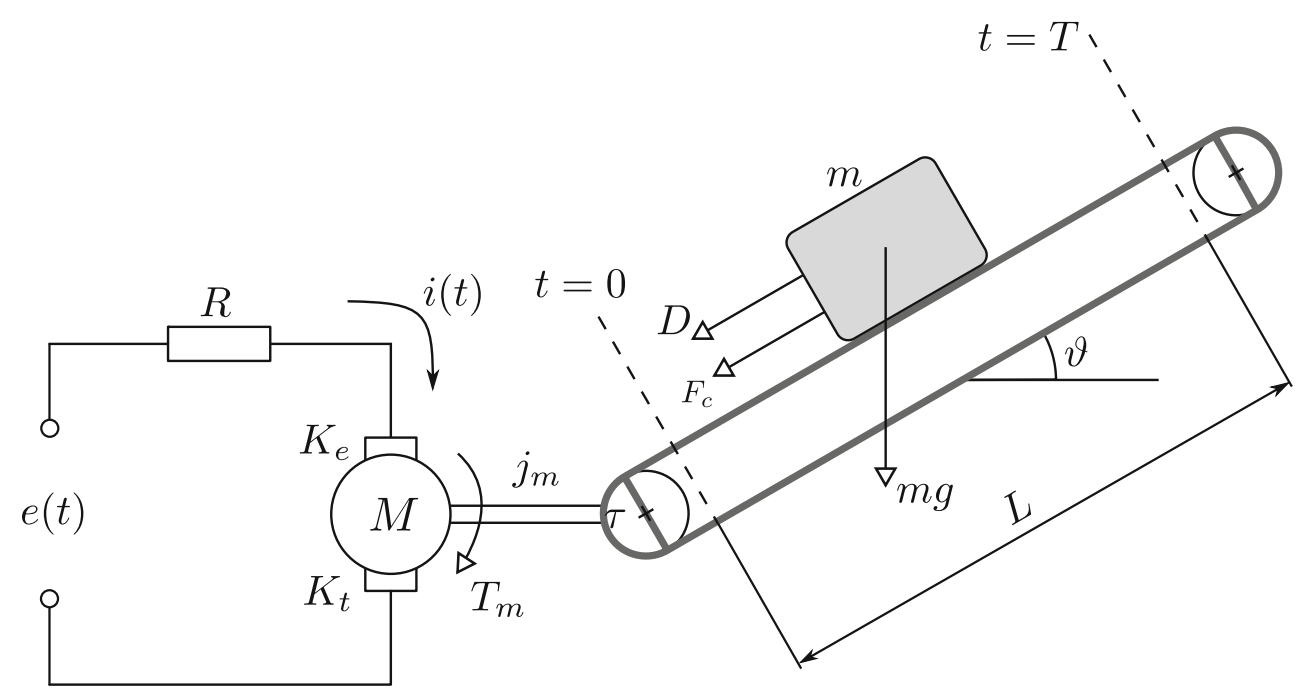

The motor torque $T_{m}$ needed to move the payload is then described by the following:

$$
\frac{T_{m}(t)}{\tau}=\left(\frac{j_{m}}{\tau^{2}}+m\right) a_{l}(t)+D v_{l}(t)+m g \sin \vartheta+F_{c}
$$

where $a_{l}(t)$ and $v_{l}(t)$ are the load acceleration and velocity, respectively, $j_{m}$ the motor moment of inertia, $m$ the load mass, $g=9.81 \mathrm{~m} / \mathrm{s}^{2}$ the gravitational acceleration, $D$ the viscous friction coefficient, and $F_{c}$ the dynamic friction. Therefore, the instantaneous current and voltage in the motor phase are:

$i(t)=\frac{T_{m}(t)}{K_{t}}=b_{1}+b_{2} v_{l}(t)+b_{3} a_{l}(t)$

$e(t)=R i(t)+\frac{K_{e}}{\tau} v_{l}(t)=b_{4}+b_{5} v_{l}(t)+b_{6} a_{l}(t)$

where $R$ is the motor winding resistance, $K_{t}$ the motor torque constant, $K_{e}$ the motor back-EMF constant, and the terms $b_{i}$ are defined as:

$$
\begin{array}{ll}
b_{1}=\left(m g \sin \vartheta+F_{c}\right) \frac{\tau}{K_{t}} & b_{4}=R b_{1} \\
b_{2}=D \frac{\tau}{K_{t}} & b_{5}=R b_{2}+\frac{K_{e}}{\tau} \\
b_{3}=\left(\frac{j_{m}}{\tau}+m \tau\right) \frac{1}{K_{t}} & b_{6}=R b_{3}
\end{array}
$$

The system consumed energy, less the recovered braking energy, is then calculated by integrating the instantaneous power $P(t)=e(t) i(t)$ over the time period $T$ :

$E=\int_{0}^{T} P(t) d t=\int_{0}^{T} e(t) i(t) d t$

In this work, it is assumed that the system is equipped with a regenerative device able to recover all the braking energy net of joule effect losses (i.e., super-capacitor with negligible losses).

\section{Energy formulation}

By substituting Eqs. 2 and 3 into Eq. 5, it holds:

$$
\begin{aligned}
E= & b_{1} b_{4} T+\left(b_{1} b_{5}+b_{2} b_{4}\right) \int_{0}^{T} v_{l}(t) d t+b_{2} b_{5} \int_{0}^{T} v_{l}(t)^{2} d t \\
& +\left(b_{1} b_{6}+b_{3} b_{4}\right) \int_{0}^{T} a_{l}(t) d t+b_{3} b_{6} \int_{0}^{T} a_{l}(t)^{2} d t \\
& +\left(b_{2} b_{6}+b_{3} b_{5}\right) \int_{0}^{T} a_{l}(t) v_{l}(t) d t
\end{aligned}
$$

Being the initial and final motion conditions known (i.e., $v(0)=0, v(T)=0, s(0)=0$, and $s(T)=L)$, the following results apply:

$\int_{0}^{T} a_{l}(t) d t=0 \quad \int_{0}^{T} v_{l}(t) d t=L$

Moreover, by integrating by parts the mixed term, it holds that:

$\int_{0}^{T} a_{l}(t) v_{l}(t) d t=\left.\frac{v_{l}(t)^{2}}{2}\right|_{0} ^{T}=0$.

The final formulation of the energy equation becomes:

$E=b_{1} b_{4} T+\left(b_{1} b_{5}+b_{2} b_{4}\right) L+b_{2} b_{5} \omega+b_{3} b_{6} \alpha$

where $\alpha=\int_{0}^{T} a_{l}(t)^{2} d t$ and $\omega=\int_{0}^{T} v_{l}(t)^{2} d t$. More in details, four terms contribute to Eq. 6 :

- A term, $b_{1} b_{4} T$, that linearly increases with the cycle time $T$ by a factor mainly dependent on the system load (i.e., with a longer cycle time, the motor has to counteract the friction and the gravity, adsorbing current and generating heat for longer).

- A constant term, $\left(b_{1} b_{5}+b_{2} b_{4}\right) L$, that depends only on system parameters and path length.

- Two motion-dependent terms, $\alpha$ and $\omega$, on which the choice of the trajectory profile and the motion primitive has effect. 
As it will be shown in the next sections, this last consideration can be helpful in the energy expenditure evaluation for a PTP motion.

\section{PTP industrial trajectory profiles}

In a PTP pick\&place task, where only the initial and final points are fixed, different trajectory profiles can be chosen. Usually, industrial motor drives implement a trapezoidal speed profile (trap) thanks to its simplicity and capability to reach low task cycle time. It is made of three motion phases (Fig. 2a), i.e., constant acceleration, constant speed, and constant deceleration. One of the main drawbacks of this motion profile regards the acceleration and deceleration phases in which the jerk reaches ideally an infinite value [38]. To avoid such a problem, modern motor drives implement a double-S or 7-segments motion profile (2s). It consists of 7 time blends (Fig. 2b), where the maximum acceleration and deceleration are gradually reached in order to have a finite jerk value. Motor drives rarely show the option to select other motion profiles and, among these, the cycloidal$S$ and polynomial profiles are here selected and considered. The cycloidal-S profile (cycl), shown in Fig. 2c, can be considered an evolution of the previous ones and it is characterized by an initial acceleration and a final deceleration phase with a cycloidal profile that leads to finite jerk values, whereas the central phase is at constant speed.

Concerning the polynomial primitives, the 3rd- (poly3), 5th- (poly5), and 7th (poly7)-order ones (Fig. 2d, e, and f, respectively) have been considered.

In Appendix A.1, the equations that define the displacement $s(t)$, velocity $v(t)$, and acceleration $a(t)$ profiles of all the considered motion primitives are reported.

\section{Energy computation and minimization}

Starting from Eq. 6, the energy formulation for each of the different trajectory profiles is obtained by computing and substituting the integral terms $\alpha$ and $\omega$ reported in Table 1. This allows evaluating the minimum energy conditions for the different trajectory profiles, compare them, and, eventually, have a closed-form solution for the problem.

\subsection{Trapezoidal speed, cycloidal-S, and double-S profiles}

In the case of trapezoidal speed, cycloidal-S, and double-S trajectory primitives, the acceleration $t_{1}$ and the deceleration $t_{3}$ times as well as the total cycle $T$ time have been selected as optimization parameters.

The time blends $t_{j a}$ and $t_{j d}$ in the double-S profile (Fig. 2b), instead, have not been considered. In fact, it can be demonstrated (Appendix A.2) that they have to be zero to minimize the consumed energy (i.e., the profile degenerates in a trapezoidal speed one). Consequently, in this work, the fixed values $t_{j a}=t_{1} / 3$ and $t_{j d}=t_{3} / 3$ have been used. Therefore, from now on, " $2 \mathrm{~s}$ " will refer to the double-S trajectory with such as assumptions.

Two different optimization cases with practical application have been then studied:

- $\quad$ 1st case: $t_{1}$ and $t_{3}$ optimized and $T$ fixed;

- 2nd case: $t_{1}, t_{3}$ and $T$ optimized.

Starting from the analytic definition of the energy Eq. 6 and considering the optimization parameters, the stationary points (i.e., minima or maxima of the function) are determined by looking at all the points in which the energy gradient is null, thus:

$$
\nabla E=\left\{\begin{array}{l}
\frac{\partial E}{\partial t_{1}}=b_{2} b_{5} \frac{\partial \omega}{\partial t_{1}}+b_{3} b_{6} \frac{\partial \alpha}{\partial t_{1}}=0 \\
\frac{\partial E}{\partial t_{3}}=b_{2} b_{5} \frac{\partial \omega}{\partial t_{3}}+b_{3} b_{6} \frac{\partial \alpha}{\partial t_{3}}=0 \\
\frac{\partial E}{\partial T}=b_{1} b_{4}+b_{2} b_{5} \frac{\partial \omega}{\partial T}+b_{3} b_{6} \frac{\partial \alpha}{\partial T}=0
\end{array}\right.
$$

where the partial derivatives of the terms $\alpha$ and $\omega$ that depend on the specific motion primitive assume the values reported in Table 2 .

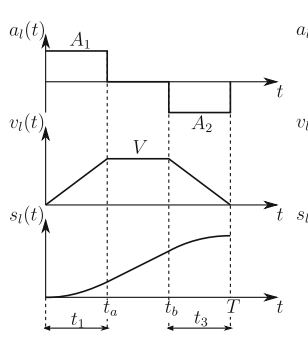

a

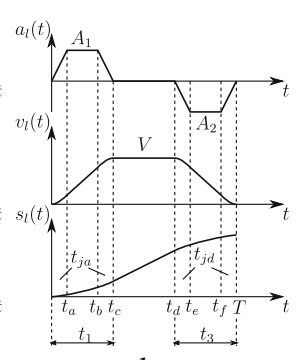

b

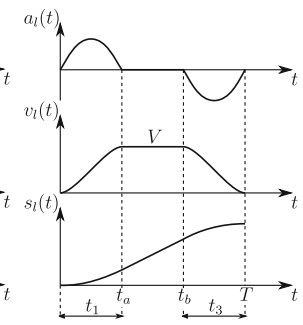

c

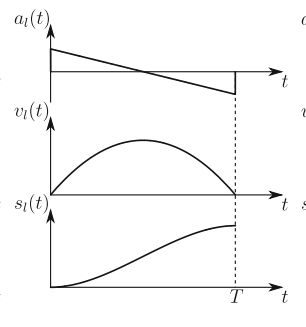

d

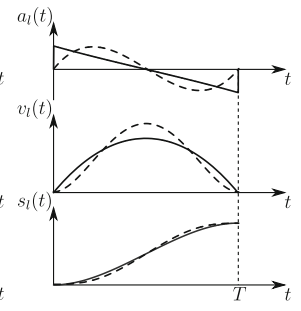

e

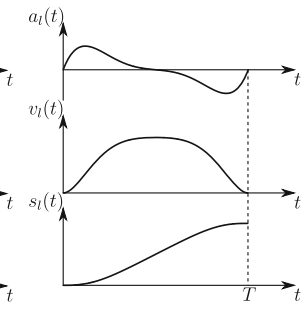

f

Fig. 2 Trajectory profiles: a trapezoidal speed profile, $\mathbf{b}$ double-S profile, $\mathbf{c}$ cycloidal-S profile, d 3rd-order polynomial profile, e 5th-order polynomial profile (continue line) and 5th-order polynomial profile with acceleration conditions (dashed line), and $\mathbf{f} 7$ th-order polynomial profile 
Table 1 Trajectory profiles integral terms

\begin{tabular}{|c|c|c|}
\hline Traj & $\alpha=\int_{0}^{T} a_{l}(t)^{2} d t$ & $\omega=\int_{0}^{T} v_{l}(t)^{2} d t$ \\
\hline trap & $\begin{array}{c}\frac{4 L^{2}\left(t_{1}+t_{3}\right)}{\left(2 T-t_{1}-t_{3}\right)^{2} t_{1} t_{3}} \\
L^{2} \pi^{2}\left(t_{1}+t_{3}\right) \\
\end{array}$ & $\frac{4 L^{2}\left(-\frac{2}{3} t_{1}+T-\frac{2}{3} t_{3}\right)}{\left(2 T-t_{1}-t_{3}\right)^{2}}$ \\
\hline $2 \mathrm{~s}$ & $\begin{array}{l}\overline{2\left(2 T-t_{1}-t_{3}\right)^{2} t_{1} t_{3}} \\
\frac{4 L^{2}}{d e n}\left(3 t_{1}^{2} t_{3}-4 t_{1}^{2} t_{j d}+3 t_{1} t_{3}^{2}-6 t_{1} t_{3} t_{j a}+\right. \\
-6 t_{1} t_{3} t_{j d}+8 t_{1} t_{j a} t_{j d}+3 t_{1} t_{j d}^{2}-4 t_{3}^{2} t_{j a}+ \\
\left.+3 t_{3} t_{j a}^{2}+8 t_{3} t_{j a} t_{j d}-4 t_{j a}^{2} t_{j d}-4 t_{j a} t_{j d}^{2}\right)\end{array}$ & $\begin{array}{l}\left(2 T-t_{1}-t_{3}\right)^{2} \\
\frac{L^{2}}{5 d e n}\left(-40 t_{1}{ }^{3} t_{3}{ }^{2}+80 t_{1}{ }^{3} t_{3} t_{j d}-40 t_{1}{ }^{3} t_{j d}{ }^{2}-40 t_{1}{ }^{2} t_{3}{ }^{3}+90 t_{1}{ }^{2} t_{3}{ }^{2} t_{j a}+\right. \\
+90 t_{1}{ }^{2} t_{3}{ }^{2} t_{j d}+60 T t_{1}{ }^{2} t_{3}{ }^{2}-180 t_{1}{ }^{2} t_{3} t_{j a} t_{j d}-65 t_{1}{ }^{2} t_{3} t_{j d^{2}}{ }^{2}+ \\
-120 T t_{1}{ }^{2} t_{3} t_{j d}+90 t_{1}{ }^{2} t_{j a} t_{j d}{ }^{2}+16 t_{1}{ }^{2} t_{j d}{ }^{3}+60 T t_{1}{ }^{2} t_{j d}{ }^{2}+ \\
+80 t_{1} t_{3}{ }^{3} t_{j a}-65 t_{1} t_{3}{ }^{2} t_{j a}{ }^{2}-180 t_{1} t_{3}{ }^{2} t_{j a} t_{j d}-120 T t_{1} t_{3}{ }^{2} t_{j a}+ \\
+130 t_{1} t_{3} t_{j a}{ }^{2} t_{j d}+130 t_{1} t_{3} t_{j a} t_{j d}{ }^{2}+240 T t_{1} t_{3} t_{j a} t_{j d}-65 t_{1} t_{j a}{ }^{2} t_{j d}{ }^{2}+ \\
-32 t_{1} t_{j a} t_{j d}{ }^{3}-120 T t_{1} t_{j a} t_{j d}{ }^{2}-40 t_{3}{ }^{3} t_{j a}{ }^{2}+16 t_{3}{ }^{2} t_{j a}{ }^{3}+ \\
+90 t_{3}{ }^{2} t_{j a}{ }^{2} t_{j d}+60 T t_{3}{ }^{2} t_{j a}{ }^{2}-32 t_{3} t_{j a}{ }^{3} t_{j d}-65 t_{3} t_{j a}{ }^{2} t_{j d}{ }^{2}+ \\
\left.-120 T t_{3} t_{j a}{ }^{2} t_{j d}+16 t_{j a}{ }^{3} t_{j d}+16 t_{j a}{ }^{2} t_{j d}{ }^{3}+60 T t_{j a}{ }^{2} t_{j d}\right)\end{array}$ \\
\hline
\end{tabular}

If the time blends $t_{j a}$ and $t_{j b}$ are fixed to $t_{j a}=t_{1} / 3$ and $t_{j d}=t_{3} / 3$ :

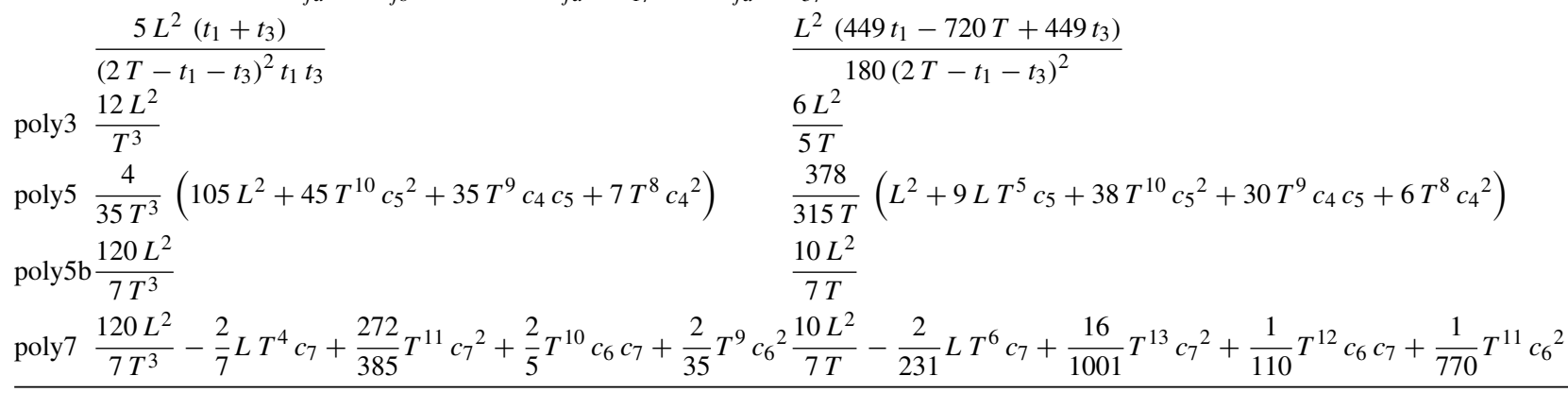

To determine whether the zeros of the functions correspond to local maxima or minima, the Hessian matrix should be considered. However, its computation is not here necessary since from Eq. 6 it could be seen that the function is convex and it has only a point of minimum since all the terms are positive.

\subsubsection{First optimization case ( $t_{1}$ and $t_{3}$ optimized, $T$ fixed)}

A closed-form solution is here found by considering the first two equations of the system (Eq. 7). Firstly, subtracting the second equation to the first:

$b_{2} b_{5}\left(\frac{\partial \omega}{\partial t_{1}}-\frac{\partial \omega}{\partial t_{3}}\right)+b_{3} b_{6}\left(\frac{\partial \alpha}{\partial t_{1}}-\frac{\partial \alpha}{\partial t_{3}}\right)=0$

and substituting the terms in Table 2, after some simplifications, and whatever motion primitive is considered (i.e., trapezoidal speed, cycloidal-S, or double-S profile), the following equation is obtained:

$\left(t_{1}^{2}-t_{3}^{2}\right)\left(2 T-t_{1}-t_{3}\right)=0$

Since $2 T-t_{1}-t_{3}$ is always positive, the condition is satisfied only when $t_{1}=t_{3}$. Therefore, the solution is determined by the first equation of system (Eq. 7), where $t_{3}=t_{1}$ and where the terms in Table 2 are substituted. After some simplifications, third-order equations can be obtained for the three trajectory profiles:

trap $:-2 t_{1}^{3}+T t_{1}^{2}+\frac{9 b_{3} b_{6}}{b_{2} b_{5}} t_{1}-\frac{3 b_{3} b_{6} T}{b_{2} b_{5}}=0$

cycl $:-5 t_{1}^{3}+3 T t_{1}^{2}+\frac{3 \pi^{2} b_{3} b_{6}}{b_{2} b_{5}} t_{1}-\frac{\pi^{2} b_{3} b_{6} T}{b_{2} b_{5}}=0$

$2 s:-449 t_{1}{ }^{3}+271 T t_{1}{ }^{2}+\frac{2700 b_{3} b_{6}}{b_{2} b_{5}} t_{1}-\frac{900 b_{3} b_{6} T}{b_{2} b_{5}}=0$

and an explicit solution can be found.

An effective way to study the energy behavior is to introduce and evaluate the term $\lambda_{1}=t_{1} / T$ into Eqs. 10, 11, and 12. Rearranging and simplifying the equations, the following "final" solutions for the trapezoidal speed, cycloidal-S, and double-S profiles, respectively, are obtained:

trap $: \frac{\left(1-2 \lambda_{1}\right) \lambda_{1}^{2}}{3\left(1-3 \lambda_{1}\right)}=\frac{b_{3} b_{6}}{T^{2} b_{2} b_{5}}$

cycl $: \frac{\left(3-5 \lambda_{1}\right) \lambda_{1}^{2}}{\pi^{2}\left(1-3 \lambda_{1}\right)}=\frac{b_{3} b_{6}}{T^{2} b_{2} b_{5}}$

$2 s: \frac{\left(271-449 \lambda_{1}\right) \lambda_{1}^{2}}{900\left(1-3 \lambda_{1}\right)}=\frac{b_{3} b_{6}}{T^{2} b_{2} b_{5}}$ 
Table 2 Gradient partial derivatives

\begin{tabular}{|c|c|c|c|c|}
\hline$\partial \alpha$ & $\begin{array}{l}\operatorname{trap} \\
4 L^{2}\left(2 t_{1}^{2}+3 t_{1} t_{3}+t_{3}^{2}-2 T t_{3}\right)\end{array}$ & $\begin{array}{l}\text { cycl } \\
L^{2} \pi^{2}\left(2 t_{1}^{2}+3 t_{1} t_{3}+t_{3}^{2}-2 T t_{3}\right)\end{array}$ & $\begin{array}{l}\mathbf{2 s} \\
5 L^{2}\left(2 t_{1}^{2}+3 t_{1} t_{3}+t_{3}^{2}-2 T t_{3}\right)\end{array}$ & \\
\hline $\begin{array}{l}\overline{\partial t_{1}} \\
\partial \alpha \\
\end{array}$ & $\begin{array}{c}t_{1}{ }^{2} t_{3}\left(2 T-t_{1}-t_{3}\right)^{3} \\
4 L^{2}\left(t_{1}^{2}+3 t_{1} t_{3}-2 T t_{1}+2 t_{3}^{2}\right)\end{array}$ & $\begin{array}{c}2 t_{1}^{2} t_{3}\left(2 T-t_{1}-t_{3}\right)^{3} \\
L^{2} \pi^{2}\left(t_{1}^{2}+3 t_{1} t_{3}-2 T t_{1}+2 t_{3}^{2}\right)\end{array}$ & $\begin{array}{c}t_{1}^{2} t_{3}\left(2 T-t_{1}-t_{3}\right)^{3} \\
5 L^{2}\left(t_{1}^{2}+3 t_{1} t_{3}-2 T t_{1}+2 t_{3}^{2}\right)\end{array}$ & \\
\hline $\begin{array}{l}\partial t_{3} \\
\partial \alpha \\
\end{array}$ & $\begin{array}{l}t_{1} t_{3}^{2}\left(2 T-t_{1}-t_{3}\right)^{3} \\
16 L^{2}\left(t_{1}+t_{3}\right) \\
\end{array}$ & $\begin{array}{r}2 t_{1} t_{3}^{2}\left(2 T-t_{1}-t_{3}\right)^{3} \\
2 L^{2} \pi^{2}\left(t_{1}+t_{3}\right) \\
\end{array}$ & $\begin{array}{l}t_{1} t_{3}^{2}\left(2 T-t_{1}-t_{3}\right)^{3} \\
20 L^{2}\left(t_{1}+t_{3}\right) \\
\end{array}$ & \\
\hline $\begin{array}{l}\partial T \\
\partial \omega\end{array}$ & $\begin{array}{l}t_{1} t_{3}\left(2 T-t_{1}-t_{3}\right)^{3} \\
8 L^{2}\left(-t_{1}+T-t_{3}\right)\end{array}$ & $\begin{array}{c}t_{1} t_{3}\left(2 T-t_{1}-t_{3}\right)^{3} \\
L^{2}\left(-5 t_{1}+6 T-5 t_{3}\right)\end{array}$ & $\begin{array}{l}t_{1} t_{3}\left(2 T-t_{1}-t_{3}\right)^{3} \\
L^{2}\left(-449 t_{1}+542 T-449 t_{3}\right)\end{array}$ & \\
\hline $\begin{array}{l}\overline{\partial t_{1}} \\
\partial \omega \\
\end{array}$ & $\begin{array}{c}3\left(2 T-t_{1}-t_{3}\right)^{3} \\
8 L^{2}\left(-t_{1}+T-t_{3}\right) \\
\end{array}$ & $\begin{array}{c}2\left(2 T-t_{1}-t_{3}\right)^{3} \\
L^{2}\left(-5 t_{1}+6 T-5 t_{3}\right) \\
\end{array}$ & $\begin{array}{c}180\left(2 T-t_{1}-t_{3}\right)^{3} \\
L^{2}\left(-449 t_{1}+542 T-449 t_{3}\right) \\
\end{array}$ & \\
\hline $\begin{array}{l}\overline{\partial t_{3}} \\
\partial \omega\end{array}$ & $\begin{array}{c}3\left(2 T-t_{1}-t_{3}\right)^{3} \\
4 L^{2}\left(5 t_{1}-6 T+5 t_{3}\right)\end{array}$ & $\begin{array}{c}2\left(2 T-t_{1}-t_{3}\right)^{3} \\
2 L^{2}\left(3 t_{1}-4 T+3 t_{3}\right)\end{array}$ & $\begin{array}{c}180\left(2 T-t_{1}-t_{3}\right)^{3} \\
L^{2}\left(269 t_{1}-360 T+269 t_{3}\right)\end{array}$ & \\
\hline$\overline{\partial T}$ & $\overline{3\left(2 T-t_{1}-t_{3}\right)^{3}}$ & $\left(2 T-t_{1}-t_{3}\right)^{3}$ & $45\left(2 T-t_{1}-t_{3}\right)^{3}$ & \\
\hline & poly3 & $\operatorname{poly5}\left(c_{i}=c_{4}, c_{j}=c_{5}\right)$ & poly5b & $\operatorname{poly7}\left(c_{i}=c_{6}, c_{j}=c_{7}\right)$ \\
\hline$\frac{\partial \alpha}{\partial c_{i}}$ & - & $4 c_{5} T^{6}+\frac{8}{5} c_{4} T^{5}$ & - & $\frac{2 T^{9}}{35}\left(2 c_{6}+7 c_{7} T\right)$ \\
\hline$\frac{\partial \alpha}{\partial c_{j}}$ & - & $\frac{72}{7} c_{5} T^{7}+4 c_{4} T^{6}$ & - & $\begin{array}{l}\frac{544}{385} c_{7} T^{11}+\frac{2}{5} c_{6} T^{10} \\
-\frac{2}{7} L T^{4}\end{array}$ \\
\hline$\frac{\partial \alpha}{\partial T}$ & $-\frac{36 L^{2}}{T^{4}}$ & $4 T^{4}\left(c_{4}+3 T c_{5}\right)^{2}-\frac{36 L^{2}}{T^{4}}$ & $-\frac{360 L^{2}}{7 T^{4}}$ & $\begin{array}{l}\frac{18 T^{8} c_{6}^{2}}{35}+4 T^{9} c_{6} c_{7}+ \\
-\frac{1800 L^{2}+40 L T^{7} c_{7}-272 T^{14} c_{7}^{2}}{35 T^{4}}\end{array}$ \\
\hline$\frac{\partial \omega}{\partial c_{i}}$ & - & $\frac{2 T^{7}}{105}\left(2 c_{4}+5 c_{5} T\right)$ & - & $\frac{T^{11}}{770}\left(2 c_{6}+7 c_{7} T\right)$ \\
\hline$\frac{\partial \omega}{\partial c_{j}}$ & - & $\frac{T^{4}}{315}\left(76 c_{5} T^{5}+30 c_{4} T^{4}+9 L\right)$ & - & $\begin{array}{l}\frac{32}{1001} c_{7} T^{13}+\frac{1}{110} c_{6} T^{12} \\
-\frac{2}{231} L T^{6}\end{array}$ \\
\hline$\frac{\partial \omega}{\partial T}$ & $-\frac{6 L^{2}}{5 T^{2}}$ & $\begin{array}{l}\frac{2 T^{6} c_{4}^{2}}{15}+\frac{16 T^{7} c_{4} c_{5}}{21}+ \\
+\frac{-126 L^{2}+12 L T^{5} c_{5}+114 T^{10} c_{5}^{2}}{105 T^{2}}\end{array}$ & $-\frac{10 L^{2}}{7 T^{2}}$ & $\begin{array}{l}\frac{T^{10} c_{6}^{2}}{70}+\frac{6 T^{11} c_{6} c_{7}}{55}+ \\
-\frac{1100 L^{2}+40 L T^{7} c_{7}-160 T^{14} c_{7}^{2}}{770 T^{2}}\end{array}$ \\
\hline
\end{tabular}

where on the right-hand side there are constant terms that do not depend on $\lambda_{1}$.

In Fig. 3, the trends for the considered trajectory profiles are shown: for each value of the constant $\frac{b_{3} b_{6}}{T^{2} b_{2} b_{5}}$ that depends on the system parameters and on the total period $T$, an optimum $\lambda_{1}$ value exists. In particular for a higher value of $\frac{b_{3} b_{6}}{T^{2} b_{2} b_{5}}$ (i.e., inertial terms have more importance), the optimum tends to $\lambda_{1}=1 / 3$ (i.e., asymptote). For values less than 0.1 (i.e., viscous terms have more importance), the optimum of $\lambda_{1}$ drops rapidly to zero.

\subsubsection{Second optimization case $\left(t_{1}, t_{3}\right.$, and $T$ optimized)}

In this second optimization case, also the third equation of the system Eq. 7 is considered. Thus, it holds:

$$
\begin{aligned}
& 3 b_{1} b_{4}\left(T-t_{1}\right)^{3} t_{1}+L^{2} b_{2} b_{5}(\left.3 T+5 t_{1}\right) t_{1}+ \\
&-12 L^{2} b_{3} b_{6}=0 \\
& 2 b_{1} b_{4}\left(T-t_{1}\right)^{3} t_{1}+L^{2} b_{2} b_{5}\left(-2 T+3 t_{1}\right) t_{1}+ \\
&-\pi^{2} L^{2} b_{3} b_{6}=0
\end{aligned}
$$

Fig. 3 First optimization case result

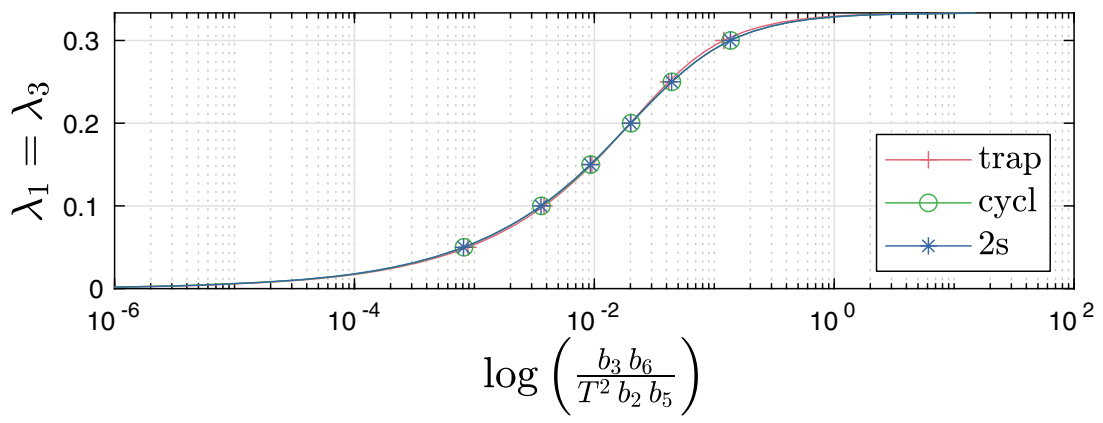




$$
\begin{gathered}
180 b_{1} b_{4}\left(T-t_{1}\right)^{3} t_{1}+ \\
+L^{2} b_{2} b_{5}\left(-180 T+269 t_{1}\right) t_{1}-900 L^{2} b_{3} b_{6}=0
\end{gathered}
$$

for the trapezoidal speed, cycloidal-S, and double-S profiles, respectively.

At the same time, the results of the previous optimization case (i.e., Eqs. 13, 14, and 15) are still true and they are used to determine the optimum $T$ value:

trap $: T=\sqrt{\frac{3 b_{3} b_{6}\left(1-3 \lambda_{1}\right)}{b_{2} b_{5} \lambda_{1}^{2}\left(1-2 \lambda_{1}\right)}}$

$c y c l: T=\sqrt{\frac{\pi^{2} b_{3} b_{6}\left(1-3 \lambda_{1}\right)}{b_{2} b_{5} \lambda_{1}^{2}\left(3-5 \lambda_{1}\right)}}$

$2 s: T=\sqrt{\frac{900 b_{3} b_{6}\left(1-3 \lambda_{1}\right)}{b_{2} b_{5} \lambda_{1}{ }^{2}\left(271-449 \lambda_{1}\right)}}$

Equations 19, 20, and 21 are then substituted into the third equation of the systems (i.e., Eqs. 16, 17, and 18), as well as $\lambda_{1}=t_{1} / T$. The following equations are thus obtained:

trap $: \frac{14 \lambda_{1}{ }^{4}-13 \lambda_{1}^{3}+3 \lambda_{1}{ }^{2}}{9\left(3 \lambda_{1}^{2}-4 \lambda_{1}+1\right)^{2}}=\frac{b_{1} b_{3} b_{4} b_{6}}{L^{2} b_{2}^{2} b_{5}^{2}}$

cycl : $\frac{20 \lambda_{1}{ }^{4}-22 \lambda_{1}{ }^{3}+6 \lambda_{1}^{2}}{4 \pi^{2}\left(3 \lambda_{1}^{2}-4 \lambda_{1}+1\right)^{2}}=\frac{b_{1} b_{3} b_{4} b_{6}}{L^{2} b_{2}^{2} b_{5}^{2}}$

$2 s: \frac{80371 \lambda_{1}^{4}-88919 \lambda_{1}^{3}+24390 \lambda_{1}^{2}}{81000\left(3 \lambda_{1}^{2}-4 \lambda_{1}+1\right)^{2}}=\frac{b_{1} b_{3} b_{4} b_{6}}{L^{2} b_{2}^{2} b_{5}^{2}}$

where on the right-hand side there are only terms that are constant and do not depend on $\lambda_{1}$.

From Eqs. 19, 20, and 21, it can be noted that values of optimum $\lambda_{1}$ higher than $1 / 3$ are not possible (i.e., the numerator of the argument of the square root becomes negative).

In Fig. 4, the trends of the functions are shown for the selected profiles: for each value of the constant $\frac{b_{1} b_{3} b_{4} b_{6}}{L^{2} b_{2}{ }^{2} b_{5}{ }^{2}}$ that depends on the system parameters, an optimum $\lambda_{1}$ value exists. Once this $\lambda_{1}$ value is determined, the optimum motion time period $T_{o p t}$ is calculated through Eqs. 19, 20, and 21 .

\subsection{Polynomial profiles}

If the polynomial trajectory profiles are focused, the optimization relies on the choice of some of their coefficients $c_{i}$ and on the total time period $T$. Similar optimization cases to the previous analysis have been considered:

- 1st case: $c_{i}$ optimized and $T$ fixed;

- 2nd case: $c_{i}$ and $T$ optimized.

Thus, the energy gradient becomes:

$\nabla E=\left\{\begin{array}{l}\frac{\partial E}{\partial c_{i}}=b_{2} b_{5} \frac{\partial \omega}{\partial c_{i}}+b_{3} b_{6} \frac{\partial \alpha}{\partial c_{i}}=0 \\ \frac{\partial E}{\partial c_{j}}=b_{2} b_{5} \frac{\partial \omega}{\partial c_{j}}+b_{3} b_{6} \frac{\partial \alpha}{\partial c_{j}}=0 \\ \frac{\partial E}{\partial T}=b_{1} b_{4}+b_{2} b_{5} \frac{\partial \omega}{\partial T}+b_{3} b_{6} \frac{\partial \alpha}{\partial T}=0\end{array}\right.$

where the partial derivatives of the terms $\alpha$ and $\omega$ that depend on the specific motion primitive assume the values as reported in Table 2.

\subsubsection{First optimization case ( $c_{i}$ optimized, $T$ fixed)}

A closed-form solution is here found by substituting in the first two equations of the system (Eq. 25) the results of Table 2. This solution exists only in the case of 5th- and 7thorder polynomial profiles, since in the other cases all the coefficients are imposed by the initial and final conditions on the speed, velocity, and, possibly, the acceleration. More in details, the following optimum results are obtained:

$$
\begin{gathered}
\text { poly5 : }\left\{\begin{aligned}
c_{4, \text { opt }} & =\frac{45 L b_{2} b_{5}}{2\left(b_{2} b_{5} T^{4}+90 b_{3} b_{6} T^{2}\right)} \\
c_{5, \text { opt }} & =-\frac{9 L b_{2} b_{5}}{b_{2} b_{5} T^{5}+90 b_{3} b_{6} T^{3}}
\end{aligned}\right. \\
\text { poly7 : }\left\{\begin{array}{l}
c_{6, \text { opt }}=-\frac{1820 L\left(b_{2} b_{5} T^{2}+33 b_{3} b_{6}\right)}{3\left(3 b_{2} b_{5} T^{8}+260 b_{3} b_{6} T^{6}\right)} \\
c_{7, o p t}=\frac{520 L\left(b_{2} b_{5} T^{2}+33 b_{3} b_{6}\right)}{3\left(3 b_{2} b_{5} T^{9}+260 b_{3} b_{6} T^{7}\right)}
\end{array}\right.
\end{gathered}
$$

Fig. 4 Second optimization case result

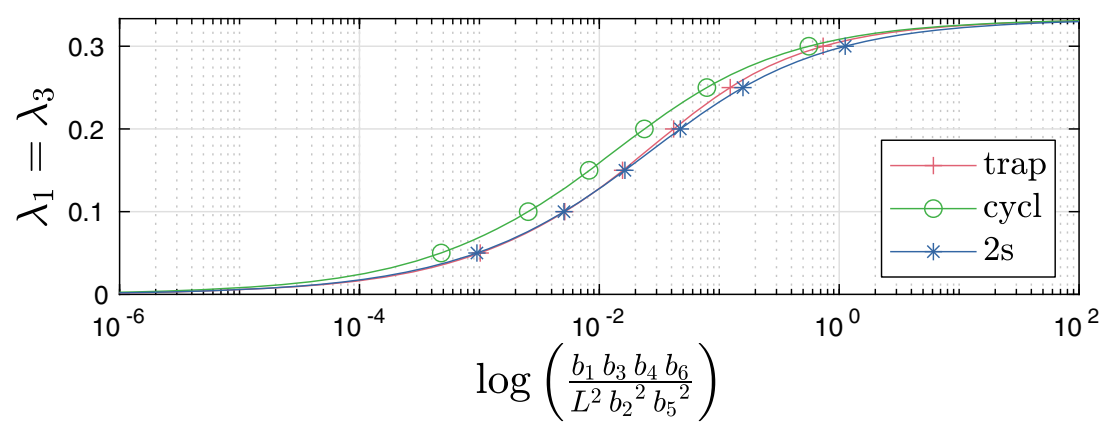




\subsubsection{Second optimization case ( $c_{i}$ and $T$ optimized)}

All the equations of the system (Eq. 25) are here considered. Focusing on the 5th- and 7th-order polynomial profiles, the solution of the first two system equations leads to the same result of Eqs. 26 and 27, respectively. Thus, substituting the gradient terms (Table 2) and Eqs. 26 and 27 into the third equation of the system (Eq. 25) allows for determining the optimum motion time period $T$ by solving:

$$
\begin{aligned}
& \text { poly5 : } 14 b_{1} b_{2}{ }^{2} b_{4} b_{5}{ }^{2} T^{8}+ \\
& +\left(2520 b_{1} b_{2} b_{3} b_{4} b_{5} b_{6}-15 L^{2} b_{2}^{3} b_{5}^{3}\right) T^{6}+ \\
& +\left(113400 b_{1} b_{3}{ }^{2} b_{4} b_{6}{ }^{2}-3690 L^{2} b_{2}{ }^{2} b_{3} b_{5}{ }^{2} b_{6}\right) T^{4}+ \\
& -226800 L^{2} b_{2} b_{3}{ }^{2} b_{5} b_{6}{ }^{2} T^{2}-4082400 L^{2} b_{3}{ }^{3} b_{6}{ }^{3}=0
\end{aligned}
$$

$$
\begin{aligned}
& \text { poly7 : 891/10 } b_{1} b_{2}^{2} b_{4} b_{5}^{2} T^{8}+ \\
& +\left(15444 b_{1} b_{3} b_{4} b_{6} b_{2} b_{5}-105 L^{2} b_{2}^{3} b_{5}^{3}\right) T^{6}+ \\
& +\left(669240 b_{1} b_{4} b_{3}^{2} b_{6}^{2}-24164 L^{2} b_{2}^{2} b_{3} b_{5}^{2} b_{6}\right) T^{4}+ \\
& -1501500 L^{2} b_{2} b_{3}^{2} b_{5} b_{6}^{2} T^{2}-28108080 L^{2} b_{3}^{3} b_{6}^{3}=0
\end{aligned}
$$

Considering the poly3 and poly $5 b$ profiles, the system (Eq. 25) comes down to a single equation (i.e., third equation of the system), since all the coefficients $c_{i}$ are defined. Substituting the terms of Table 2, the optimum motion time results:

poly3 $: T=\sqrt{\frac{L \sqrt{L^{2} b_{2}^{2} b_{5}^{2}+100 b_{1} b_{3} b_{4} b_{6}}+L^{2} b_{2} b_{5}}{5 / 3 b_{1} b_{4}}}$

poly5 :

$$
T=\sqrt{\frac{\sqrt{5} L \sqrt{5 L^{2} b_{2}^{2} b_{5}^{2}+504 b_{1} b_{3} b_{4} b_{6}}+5 L^{2} b_{2} b_{5}}{7 b_{1} b_{4}}}
$$

\section{Energy comparison}

In this section, the different trajectories are compared in terms of energy expenditure. In order to do so, they are supposed to be applied on the same mechatronic system moving the same load on the same path and in the same cycle time. In addition, the optimum parameters of each motion primitive that minimizes the energy consumption are considered. In this way, the problem of how to treat equations with dissimilar parameters (e.g., the trapezoidal speed trajectory is defined by the acceleration and deceleration times $t_{1}$ and $t_{3}$, whereas polynomial trajectories are defined by the polynomial coefficients $c_{i}$ ) is overcome.
Starting from the general energy equation (Eq. 6), the contribution of the two terms $b_{1} b_{4} T$ and $\left(b_{1} b_{5}+b_{2} b_{4}\right) L$ can be ignored for the comparison purpose, since they are always in common. Moreover, the integral terms $\alpha$ and $\omega$ are calculated by substituting the optimum solution of each trajectory profile (Table 1 ), obtaining $\alpha_{o p t}$ and $\omega_{\text {opt }}$ (Table 3). Therefore:

$$
E^{I}=b_{2} b_{5} \omega_{o p t}+b_{3} b_{6} \alpha_{o p t}
$$

Collecting and highlighting the common terms (Table 3), the energy equation can be rewritten as:

$$
E^{I I}=b_{2} b_{5} \frac{L^{2}}{T} f_{\omega}+b_{3} b_{6} \frac{L^{2}}{T^{3}} f_{\alpha}
$$

where $f_{\omega}=\frac{T}{L^{2}} \omega_{o p t}$ and $f_{\alpha}=\frac{T^{3}}{L^{2}} \alpha_{o p t}$. Finally, dividing by $b_{2} b_{5} L^{2} / T$, it results:

$E^{I I I}=f_{\omega}+\frac{b_{3} b_{6}}{T^{2} b_{2} b_{5}} f_{\alpha}$

The difference in energy consumption between the motion primitives depends, thus, only on the constant $\frac{b_{3} b_{6}}{T^{2} b_{2} b_{5}}$ (i.e., $b_{i}$ and $T$ are assumed fixed) and the two terms $f_{\alpha}$ and $f_{\omega}$. Note that these terms depend in turn on the same constant $\frac{b_{3} b_{6}}{T^{2} b_{2} b_{5}}$ (Table 3$)$.

In Fig. 5, the ratio of $E_{i}^{I I I}$, with $i=\operatorname{trap}$, cycl, 2s, poly3, poly5, poly5b, and poly7, over $E_{\text {trap }}^{I I I}$ is computed in function of $\frac{b_{3} b_{6}}{T^{2} b_{2} b_{5}}$. Different behaviors occur depending on the $\frac{b_{3} b_{6}}{T^{2} b_{2} b_{5}}$ value. As a first result, the 5 th-order polynomial trajectory with imposed initial and final acceleration (i.e., poly5b) is always more energy-intensive than the others. It consumes up to more than the $40 \%$ of the part of energy related to the motion primitive, with respect to the trapezoidal speed profile, when $\frac{b_{3} b_{6}}{T^{2} b_{2} b_{5}}$ is small. The trapezoidal speed profile results the more efficient when $\frac{b_{3} b_{6}}{T^{2} b_{2} b_{5}}<0.001$. For higher values of $\frac{b_{3} b_{6}}{T^{2} b_{2} b_{5}}$, the 5thorder polynomial and the 3rd-order polynomial have a better behavior (i.e., they consume about the $10 \%$ less).

\section{Numerical evaluation}

A hoist mechanism, visible in Fig. 6, is considered as a test-case for applying the developed method.

It consists on a motor coupled with a planetary gear-train that drives a spool to lift a suspended weight. In addition, the system is able to recover the braking energy and store it in the drive capacitors. Referring to Section 2, and in particular to Fig. 1, the following considerations are done: $\vartheta=\pi / 2$ and $\tau=\tau^{\prime} d / 2$ with $\tau^{\prime}$ the transmission ratio of the gearset and $d$ the spool diameter. Moreover, the equivalent inertia 
Table 3 Optimized trajectory profiles integral terms

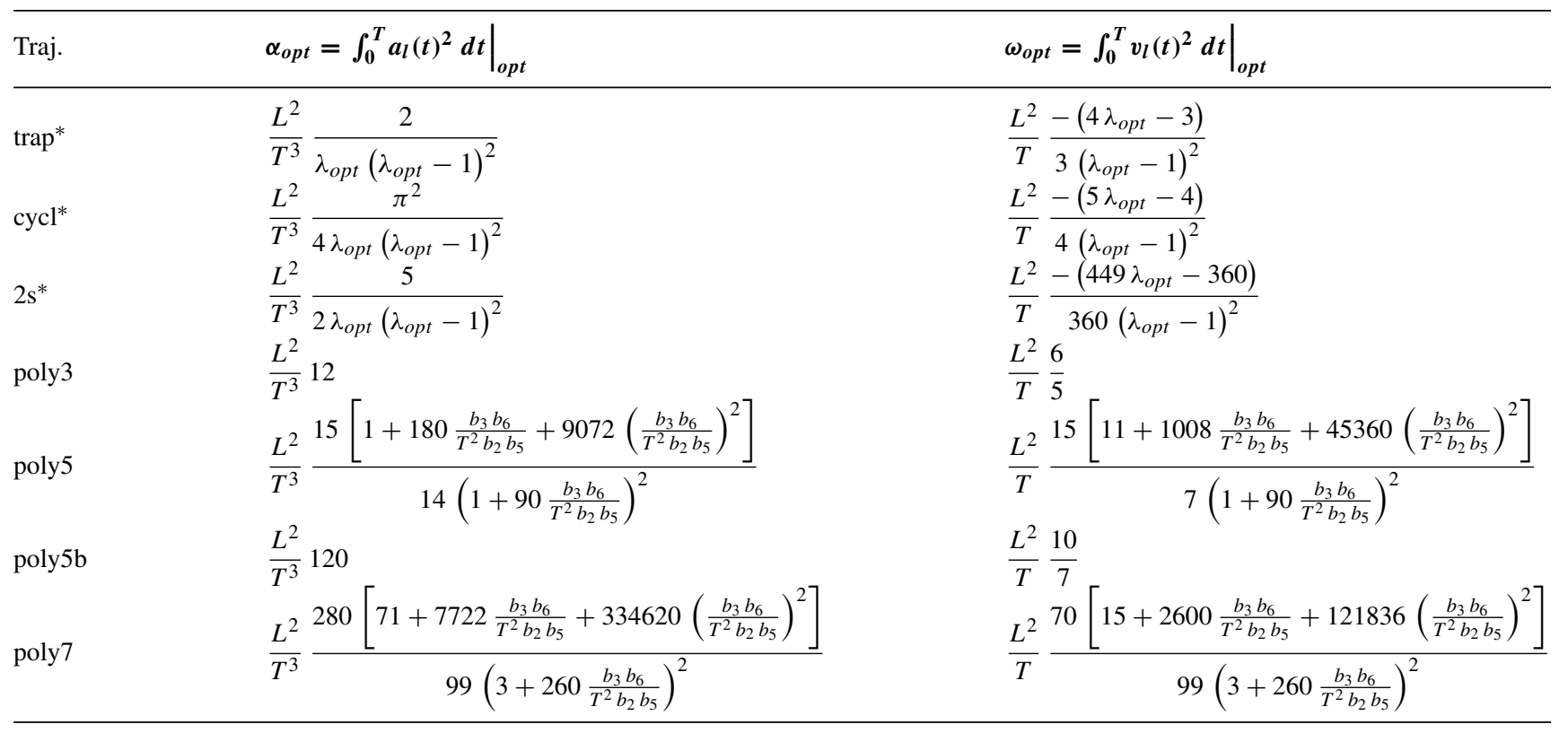

${ }^{*} \lambda_{\text {opt }}=f\left(\frac{b_{3} b_{6}}{T^{2} b_{2} b_{5}}\right)$.

of the spool (i.e., $j_{l} \tau^{\prime 2}$ ) is considered within the motor inertia $j_{m}$. Table 4 summarizes all the system parameters in accordance with Fig. 2.

In this study, only the ascend motion of the load is studied. Anyway, the same approach and analogous results can be retrieved for the descend movement.

By applying the optimization approach through the equations derived in Section 5 for all the motion primitives, the results in Table 5 are obtained.

Considering the first optimization case, where a motion time period $T=4 \mathrm{~s}$ is selected and $\frac{b_{3} b_{6}}{T^{2} b_{2} b_{5}}=8.893 \cdot 10^{-5}$, it can be noted how the optimum acceleration/deceleration times (i.e., $\lambda_{1}$ ) are at relatively short time periods and, thus,

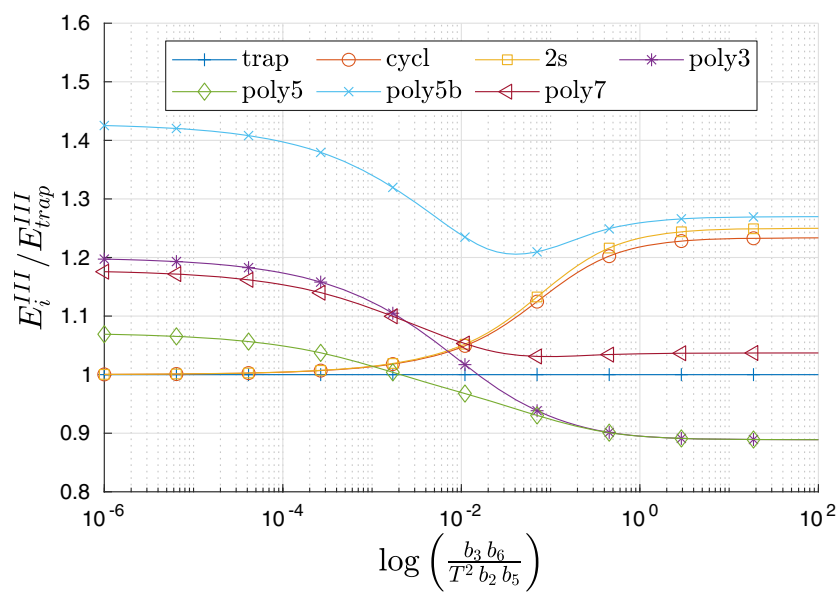

Fig. 5 Comparison between consumed energy by a generic mechatronic system using different trajectories at the left side in Fig. 3 in the case of non-polynomial profiles. This could represent a problem in some systems, since high torque peaks are required to run the trajectory and they could not be feasible. In the second optimization case where $\frac{b_{1} b_{3} b_{4} b_{6}}{L^{2} b_{2}^{2} b_{5}^{2}}=2.894 \cdot 10^{-4}$, the computed optimum motion time period $T$ is between 1.67 and $2.66 s$, depending on the selected trajectory. Again, the computed

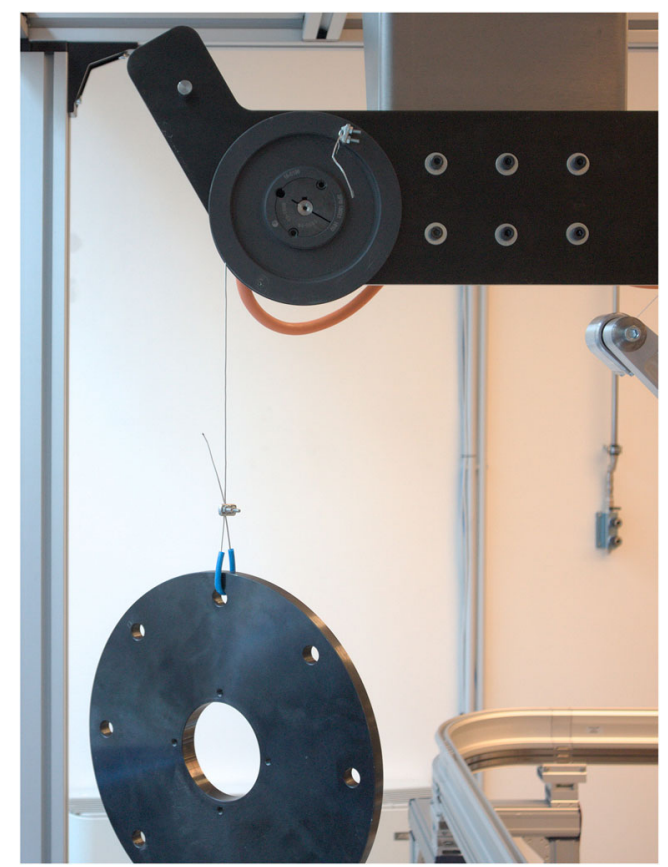

Fig. 6 Hoist system 
Table 4 Test-case parameters

\begin{tabular}{llll}
\hline Symbol & Value & Symbol & Value \\
\hline$d$ & $0.157 \mathrm{~m}$ & $K_{t}$ & $0.85 \mathrm{Nm} / \mathrm{A}$ \\
$L$ & $\pi d$ & $K_{e}$ & $0.85 \mathrm{~V} \mathrm{~s} / \mathrm{rad}$ \\
$m$ & $11.83 \mathrm{~kg}$ & $R$ & $10.7 \Omega$ \\
$\tau$ & $1 / 35$ & $D$ & $0.0611 \mathrm{Ns} / \mathrm{m}$ \\
$j_{m}$ & $55.34 \cdot 10^{-6} \mathrm{~kg} \mathrm{~m}^{2}$ & $F_{c}$ & $18.58 \mathrm{~N}$ \\
\hline
\end{tabular}

acceleration/deceleration times are very short (i.e., left side in Fig. 4 in the case of non-polynomial profiles).

\section{Experimental evaluation}

The hoist system has been used also as an experimental test-case in order to finally validate the method and the numerical results. A real system (Fig. 6) has been realized in the Smart Mini Factory of the Free University of BozenBolzano. A Kollmorgen AKM31C PMSM motor coupled with a Beckhoff AG3210-NP015S planetary gearset with a $1 / 35$ transmission ratio are used to move the spool. The motor is controlled in position by a Kollmorgen AKDP00307 servo-drive that is able to reproduce any motion profile defined through a table. A braking energy recovering system, integrated in the drive, is able to store up to $9 \mathrm{~J}$ of energy. A series of Matlab scripts and functions have been created in order to communicate with the drive and program it. In particular, the connection is based on a telnet protocol and established on a LAN network. The drive is able to acquire and save internal parameters (e.g., speed, current, DC bus voltage) with its built-in scope function. A Matlab function has thus been created to manage the scope function and acquire the instantaneous speed $n$ and the current qaxis component $I_{q}$, proportional to the output torque, with a sampling frequency of $1 \mathrm{kHz}$. The scope function has been

Table 5 Test-case numerical results (a motion time period $T$ of $4 s$ has been considered in the 1st optimization case)

\begin{tabular}{llllllll}
\hline \multicolumn{7}{c}{ 1st opt. case } & 2nd opt. case \\
\hline Traj. & $\lambda_{1, \text { opt }}$ & & $E[J]$ & $T_{\text {opt }}[s]$ & $\lambda_{1, \text { opt }}$ & $E[J]$ \\
trap & 0.0162 & & 73.8 & 2.30 & 0.0279 & 72.8 \\
cycl & 0.0169 & & 73.8 & 1.67 & 0.0398 & 73.2 \\
2s & 0.0170 & & 73.8 & 2.32 & 0.0290 & 72.8 \\
Traj. & $c_{i, o p t}$ & $c_{j, o p t}$ & $E[J]$ & $T_{o p t}[s]$ & $c_{i, o p t}$ & $c_{j, o p t}$ & $E[J]$ \\
poly3 & - & - & 74.1 & 2.44 & - & - & 73.2 \\
poly5 & 0.0430 & -0.0043 & 73.9 & 2.31 & 0.3775 & -0.0043 & 72.9 \\
poly5b & - & - & 74.4 & 2.66 & - & - & 73.8 \\
poly7 & -0.0242 & 0.0017 & 74.0 & 2.42 & -0.4861 & 0.0017 & 73.2 \\
\hline
\end{tabular}

exploited to measure and calculate the energy consumed by the system performing all the different trajectories. Firstly, the mechanical power is calculated as:

$P_{m}=\frac{2 \pi n}{60} K_{t} I_{q}$

The power lost as heat in the motor wound is instead computed as:

$P_{j}=R I^{2}=R \frac{3}{2} \sqrt{I_{q}^{2}+I_{d}^{2}} \simeq \frac{3}{2} R I_{q}^{2}$

where the last assumptions consider that the direct-axis current $I_{d}$ is always zero for this kind of machine and in the considered working zones (i.e., flux weakening not used since high speeds are not reached). The coefficient $3 / 2$ is due since the drive uses the formal Clark transformation [39]. The electrical energy is then computed as $E=$ $\int_{0}^{T} P_{m}+P_{j} d t$.

The system parameters are the same reported in Table 4. On this regard, all of them have been determined by collecting data from a data-sheet, except for the friction terms $F_{c}$ and $D$ that have been instead retrieved experimentally.

In this experimental campaign, all the seven trajectory profiles have been tested, considering different trajectory parameters. For what concerns the first three profiles (i.e., trapezoidal speed, cycloidal, and double-S profiles), the energy consumption is measured in experiments where both the time period $T$ and the acceleration/deceleration time $t_{1}=t_{3}$ are varied. Note that the optimum $\lambda_{1}$ values reported in Table 5 cannot be achieved by the experimental system (i.e., the rope can work only in tension; thus, deceleration higher than $9.81 \mathrm{~m} / \mathrm{s}^{2}$ is not admissible). Anyway, a closer value, $\lambda=0.1$, is reachable.

In the case of polynomial profiles, the influence of the time period $T$ on the energy consumption has been investigated. In the case of 5 th and 7 th degree polynomial profiles, two of the polynomial profile coefficients are free to be chosen; in these experiments, only the optimum coefficients (i.e., Table 5) that maximize the energy efficiency have been considered. Each test has been repeated 5 times, as shown in Fig. 7.

The results of the experimental campaign are summarized in Fig. 8a where the trajectories are all compared together.

As foreseen by the model and the numerical evaluation (Table 5), the minimum energy consumption point falls in a range between 2 and $3 s$ for all the curves. Then, the energy increases for shorter and longer time periods (i.e., convex shape). As expected from the theory (Section 6), the trapezoidal speed profile results the less energy-intensive, since the system constant $\frac{b_{3} b_{6}}{T^{2} b_{2} b_{5}}<0.001$. More in general, the energy consumption rank of all the motion primitives follows the Fig. 5 prediction. 
Fig. 7 Comparison between experimental data and numerical model in the case of the trapezoidal speed profile with $\lambda=0.1$

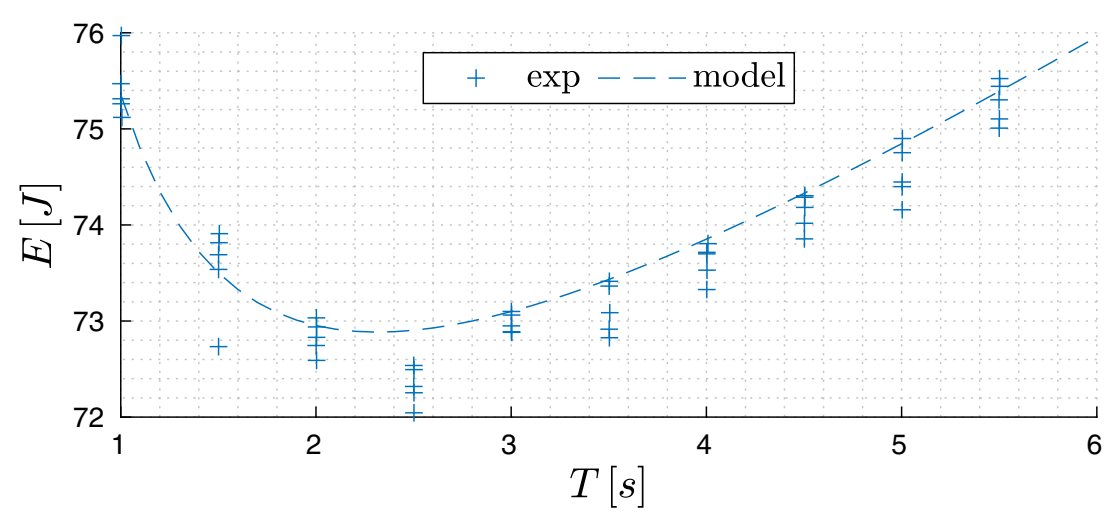

In Fig. 8b, the influence of the acceleration and deceleration time is shown in the case of a trapezoidal speed profile, demonstrating that in this system lower values of $t_{1}=t_{3}$ lead to a minimum of the energy consumption. For instance, an energy reduction of about $1.3 \%$ can be achieved if $t_{1}=$ $t_{3}=1 / 10$, instead of using the most common $1 / 3$ values (i.e., the energy from $73.41 J$ falls down to $72.43 J$, considering the time period $T=2.5 \mathrm{~s}$ ), and this while maintaining the same productivity (i.e., $T$ does not change). Analogous

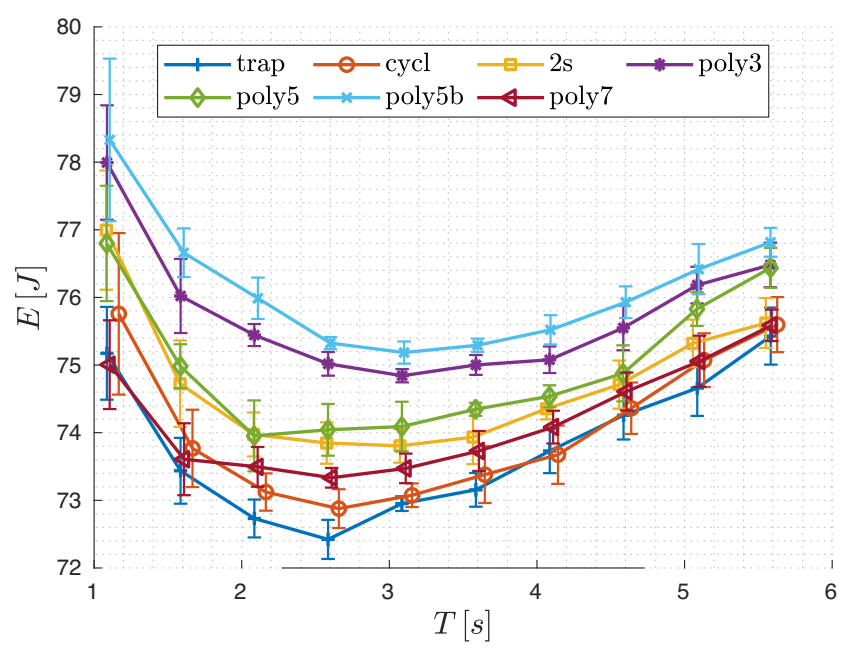

a

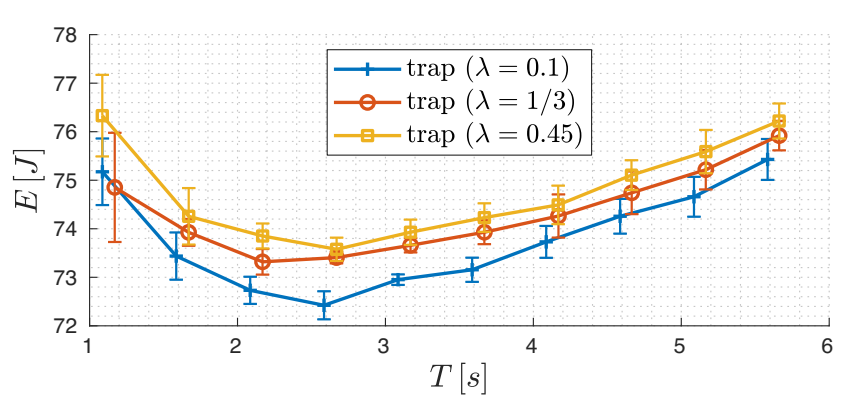

$\mathrm{b}$

Fig. 8 Experimental results: a energy consumption comparison between the different trajectory profiles; $\mathbf{b}$ trapezoidal speed profile (note that each data point is the mean value of five tests) results can be obtained considering the cycloidal-S and the double-S profiles.

\section{Conclusion}

In this paper, the study of the energy consumption and its minimization in generic $1-\mathrm{DoF}$ mechatronic systems moving a constant inertia load (e.g., elevators, cranes, $\mathrm{CNC}$ machines, Cartesian axis) and possibly equipped or retrofitted with regenerative devices (i.e., mixed approach) has been done. Different PTP trajectory profiles have been investigated including both profiles commonly used in industrial motor drives and more complex ones.

In particular, the function of energy consumption has been analytically expressed finding out that it depends on six constant $b_{i}$ terms that describe the system parameters (e.g., mass, inertia, friction), the length of the motion path $L$, the total time period $T$, and the speed and acceleration of the motion profiles. Then, the developed approach considers the analytical energetic optimization of all the different PTP primitives by deriving the optimum closed-form solutions.

For what concerns the standard trajectories (i.e., trapezoidal speed and double-S profiles), as well as the cycloidal one, the optimization has been achieved by varying the total motion time $T$ and the acceleration and deceleration time periods. Conversely, in the case of polynomial primitives, the motion time period $T$ has been optimized, as well as the polynomial coefficients that have effect on the "shape" of the profile. The energy function presents a convex trend with respect to the total motion time $T$ and, thus, a unique $T$ that guarantees the minimum energy consumption exists.

In addition to similar works, it has been demonstrated and verified that, under the considered hypothesis and working conditions, the optimum solution is the one obtained when the same values for the acceleration and deceleration time periods are chosen (i.e., $\lambda_{1}=\lambda_{3}$ ). The effect of changing the $\lambda_{i}$ values on the overall energy expenditure (i.e., from $\lambda=0.1$ to $\lambda=0.5$ ), even if is less heavy if compared with the variation of $T$, is higher in systems in which the inertial terms 
are greater than the effect of external constant force/torque. This numerical result shows as, in particular in repetitive tasks, the saved energy could reach substantial values.

Considering a practical application, this analytical approach allows for a fast and easy computation to determine the point of minimum energy. Therefore, it is worth to be integrated in (existing) embedded mechatronic control systems, where computational power is limited, for online trajectory optimization. Even if some constraints (e.g., maximum motor current) are not directly taken into account, the analytical solution can be used as a starting point for a numerical method, where only the motion-dependent terms are minimized as discussed in Section 3 (i.e., less computation efforts). Anyway, in future studies, the introduction of system constraints into the analytical formulation will be valuable, to further speed-up the computation of the optimal trajectory. Other motion primitives will be also investigated (e.g., Gutman, Freudenstein).

\section{Appendix}

\section{A.1 PTP industrial trajectory profiles}

In this section, the equations that define all the considered trajectory profiles are reported. In particular, with reference to Fig. 2, the displacement $s_{l}(t)$, the velocity $v_{l}(t)$, and the acceleration $a_{l}(t)$ of the load are defined.

\section{A.1.1 Trapezoidal speed profile}

$s_{l}(t)=\left\{\begin{array}{lr}\frac{1}{2} A_{1} t^{2} & t \in\left[0, t_{a}\right] \\ s_{a}+A_{1} t_{a}\left(t-t_{a}\right) & \left.t \in] t_{a}, t_{b}\right] \\ s_{b}+A_{1} t_{a} t+A_{2} t_{b} t-\frac{1}{2} A_{2} t^{2} & \left.t \in] t_{b}, T\right]\end{array}\right.$

$v_{l}(t)= \begin{cases}A_{1} t & t \in\left[0, t_{a}\right] \\ A_{1} t_{a} & \left.t \in] t_{a}, t_{b}\right] \\ A_{1} t_{a}-A_{2}\left(t-t_{b}\right) & \left.t \in] t_{b}, T\right]\end{cases}$

$a_{l}(t)= \begin{cases}A_{1} & t \in\left[0, t_{a}\right] \\ 0 & \left.t \in] t_{a}, t_{b}\right] \\ -A_{2} & \left.t \in] t_{b}, T\right]\end{cases}$

where $s_{i}=s\left(t_{i}\right)$ and the constants $A_{1}$ and $A_{2}$ are retrieved by imposing the total path length (i.e., $s(T)=L$ ) and the equality between the initial and final speed of constant speed part (i.e., $\left.V=v\left(t_{a}\right)=v\left(t_{b}\right)\right): A_{1}=\frac{2 L}{\left(2 T-t_{1}-t_{3}\right) t_{1}}$ and $A_{2}=A_{1} \frac{t_{1}}{t_{3}}$.

\section{A.1.2 Cycloidal-S profile}

$s_{l}(t)= \begin{cases}\frac{V}{2}\left(t-\frac{\sin \left(\omega_{1} t\right)}{\omega_{1}}\right) & t \in\left[0, t_{a}\right] \\ V\left(t-\frac{t_{1}}{2}\right) & \left.t \in] t_{a}, t_{b}\right] \\ V\left(T-\frac{t_{1}}{2}-t_{3}\right)+\frac{V}{2}\left(t^{\prime}+\frac{\sin \left(\omega_{3} t^{\prime}\right)}{\omega_{3}}\right) & \left.t \in] t_{b}, T\right]\end{cases}$ $v_{l}(t)= \begin{cases}\frac{V}{2}\left(1-\cos \left(\omega_{1} t\right)\right) & t \in\left[0, t_{a}\right] \\ V & \left.t \in] t_{a}, t_{b}\right] \\ \frac{V}{2}\left(1+\cos \left(\omega_{3} t^{\prime}\right)\right) & \left.t \in] t_{b}, T\right]\end{cases}$

$a_{l}(t)= \begin{cases}\frac{V}{2} \omega_{1} \sin \left(\omega_{1} t\right) & t \in\left[0, t_{a}\right] \\ 0 & \left.t \in] t_{a}, t_{b}\right] \\ -\frac{V}{2} \omega_{3} \sin \left(\omega_{3} t^{\prime}\right) & \left.t \in] t_{b}, T\right]\end{cases}$

where $\omega_{1}=\frac{\pi}{t_{1}}, \omega_{3}=\frac{\pi}{t_{3}}$, and $t^{\prime}=t-T+t_{3}$. The constant $V$ is derived by imposing the path length (i.e., $s(T)=L$ ): $V=\frac{2 L}{2 T-t_{1}-t_{3}}$.

\section{A.1.3 Double-S profile}

$s_{l}(t)= \begin{cases}\frac{1}{6} \frac{A_{1}}{t_{j 1}} t^{3} & t \in\left[0, t_{a}\right] \\ s_{a}+v_{a}\left(t-t_{a}\right)+\frac{1}{2} A_{1}\left(t-t_{a}\right)^{2} & \left.t \in] t_{a}, t_{b}\right] \\ s_{b}+v_{b}\left(t-t_{b}\right)+\frac{A_{1}\left(t-t_{b}\right)^{2}}{2}\left(1-\frac{t-t_{b}}{3 t_{j 2}}\right) & \left.t \in] t_{b}, t_{c}\right] \\ s_{c}+v_{c}\left(t-t_{c}\right) & \left.t \in] t_{c}, t_{d}\right] \\ s_{d}+v_{d}\left(t-t_{d}\right)-\frac{1}{6} A_{2} \frac{\left(t-t_{d}\right)^{3}}{t_{j 3}} & \left.t \in] t_{d}, t_{e}\right] \\ s_{e}+v_{e}\left(t-t_{e}\right)-\frac{1}{2} A_{2}\left(t-t_{e}\right)^{2} & \left.t \in] t_{e}, t_{f}\right] \\ s_{f}+v_{f}\left(t-t_{f}\right)-\frac{A_{2}\left(t-t_{f}\right)^{2}}{2}\left(1-\frac{t-t_{f}}{3 t_{j 4}}\right) & \left.t \in] t_{f}, T\right]\end{cases}$

$$
\begin{aligned}
& v_{l}(t)= \begin{cases}\frac{1}{2} \frac{A_{1}}{t_{j 1}} t^{2} & t \in\left[0, t_{a}\right] \\
v_{a}+A_{1}\left(t-t_{a}\right) & \left.t \in] t_{a}, t_{b}\right] \\
v_{b}+A_{1}\left[t-t_{b}-\frac{\left(t-t_{b}\right)^{2}}{2 t_{j 2}}\right] & \left.t \in] t_{b}, t_{c}\right] \\
v_{c} & \left.t \in] t_{c}, t_{d}\right] \\
v_{d}-\frac{1}{2} A_{2} \frac{\left(t-t_{d}\right)^{2}}{t_{j 3}} & \left.t \in] t_{d}, t_{e}\right] \\
v_{e}-A_{2}\left(t-t_{e}\right) & \left.t \in] t_{e}, t_{f}\right] \\
v_{f}-A_{2}\left[t-t_{f}-\frac{\left(t-t_{f}\right)^{2}}{2 t_{j 4}}\right] & \left.t \in] t_{f}, T\right]\end{cases} \\
& a_{l}(t)= \begin{cases}\frac{A_{1}}{t_{j 1}} t & t \in\left[0, t_{a}\right] \\
A_{1} & \left.t \in] t_{a}, t_{b}\right] \\
A_{1}\left(1-\frac{t-t_{b}}{t_{j 2}}\right) & \left.t \in] t_{b}, t_{c}\right] \\
0 & \left.t \in] t_{c}, t_{d}\right] \\
-A_{2} \frac{t-t_{d}}{t_{j 3}} & \left.t \in] t_{d}, t_{e}\right] \\
-A_{2} & \left.t \in] t_{e}, t_{f}\right] \\
-A_{2}\left(1-\frac{t-t_{f}}{t_{j 4}}\right) & \left.t \in] t_{f}, T\right]\end{cases}
\end{aligned}
$$

where $s_{i}=s\left(t_{i}\right), v_{i}=v\left(t_{i}\right)$, and the acceleration constants $A_{1}$ and $A_{2}$ are retrieved by imposing the total path length (i.e., $s(T)=L$ ) and $V=v\left(t_{a}\right)=v\left(t_{b}\right)$. In this work, as done in most of the practical applications, the acceleration ramp times (i.e., $t_{j 1}=t_{j 2}=t_{j a}$ ) as well as the deceleration ramp times (i.e., $t_{j 3}=t_{j 4}=t_{j d}$ ) are considered equal. In this way, $A_{1}=-\frac{2 L}{\left(t_{1}-t_{j a}\right)\left(t_{1}-2 T+t_{3}\right)}$ and $A_{2}=A_{1} \frac{t_{1}-t_{j a}}{t_{3}-t_{j d}}$.

\section{A.1.4 3rd-order polynomial profile}

$s_{l}(t)=c_{0}+c_{1} t+c_{2} t^{2}+c_{3} t^{3}$ 
$v_{l}(t)=c_{1}+2 c_{2} t+3 c_{3} t^{2}$

$a_{l}(t)=2 c_{2}+6 c_{3} t$

where $c_{0}=0, c_{1}=0, c_{2}=\frac{3 L}{T^{2}}$, and $c_{3}=-\frac{2 L}{T^{3}}$ are the polynomial coefficients which values are given by imposing the initial conditions on displacement (i.e., $s_{l}(0)=0$ and $s_{l}(T)=L$ ) and velocity (i.e., $v_{l}(0)=0$ and $v_{l}(T)=0$ ).

\section{A.1.5 5th-order polynomial profile}

$s_{l}(t)=c_{0}+c_{1} t+c_{2} t^{2}+c_{3} t^{3}+c_{4} t^{4}+c_{5} t^{5}$

$v_{l}(t)=c_{1}+2 c_{2} t+3 c_{3} t^{2}+4 c_{4} t^{3}+5 c_{5} t^{4}$

$a_{l}(t)=2 c_{2}+6 c_{3} t+12 c_{4} t^{2}+20 c_{5} t^{3}$

where $c_{0}=0, c_{1}=0, c_{2}=\frac{3 L}{T^{2}}+c_{4} T^{2}+2 c_{5} T^{3}$, and $c_{3}=c_{3}=-\frac{2 L}{T^{3}}-2 c_{4} T-3 c_{5} T^{2}$ are the polynomial coefficients which values are computed by imposing the initial conditions on displacement (i.e., $s_{l}(0)=0$ and $s_{l}(T)=L$ ) and velocity (i.e., $v_{l}(0)=0$ and $v_{l}(T)=0$ ). The values of $c_{4}$ and $c_{5}$ can be freely chosen or via, e.g., a minimum energy criterion.

If also the initial and final accelerations are imposed (i.e., $a_{l}(0)=0$ and $a_{l}(T)=0$ ), it results: $c_{0}=0, c_{1}=0, c_{2}=0$, $c_{3}=\frac{10 L}{T^{3}}, c_{4}=-\frac{15 L}{T^{4}}$, and $c_{5}=\frac{6 L}{T^{5}}$. In this case, we refer to this trajectory with the name poly $5 b$.

\section{A.2 Double-S profile optimal time blends}

For the double-S profile, the resolution of the optimum problem is not immediate and a closed-form is hard to find. Anyway, thanks to some considerations and simplifications, the terms in Table 2 can be computed. It is also possible to demonstrate that the time blends $t_{j a}$ and $t_{j d}$ should be zero to minimize the consumed energy and thus the Double$S$ profile degenerates in a trapezoidal speed profile. Indeed, considering the partial derivatives:

$$
\begin{aligned}
\frac{\partial \alpha_{2 s}}{\partial t_{j a}} & =\frac{8 L^{2}\left(t_{1}-2 t_{j a}\right)}{3\left(t_{1}-t_{j a}\right)^{3}\left(t_{1}-2 T+t_{3}\right)^{2}} \\
\frac{\partial \omega_{2 s}}{\partial t_{j a}} & =\frac{2 L^{2}\left(5 t_{1}^{3}-20 t_{1}^{2} t_{j a}+24 t_{1} t_{j a}{ }^{2}-8 t_{j a}{ }^{3}\right)}{15\left(t_{1}-t_{j a}\right)^{3}\left(t_{1}-2 T+t_{3}\right)^{2}}
\end{aligned}
$$

they are always positive for $0 \leq t_{j a} \leq t_{1} / 2$; thus, the functions $\alpha$ and $\omega$ are both minimized and thus the energy, when $t_{j a}$ is zero. The same conclusion can be obtained for $t_{j d}$ that has to be zero to minimize the consumed energy. In this way, $t_{j a}$ and $t_{j b}$ can be seen as parameters and not variables.
Author contribution All authors contributed equally to this manuscript.

Funding Open access funding provided by Libera Università di Bolzano within the CRUI-CARE Agreement. This work was partially supported by the Free University of Bozen-Bolzano funds within the project TN2803: "Mech4SME3: Mechatronics for Smart Maintenance and Energy Efficiency Enhancement.”

\section{Declarations}

Consent to publish All authors agree to transfer copyright of this article to the publisher.

Competing interests The authors declare no competing interests.

Open Access This article is licensed under a Creative Commons Attribution 4.0 International License, which permits use, sharing, adaptation, distribution and reproduction in any medium or format, as long as you give appropriate credit to the original author(s) and the source, provide a link to the Creative Commons licence, and indicate if changes were made. The images or other third party material in this article are included in the article's Creative Commons licence, unless indicated otherwise in a credit line to the material. If material is not included in the article's Creative Commons licence and your intended use is not permitted by statutory regulation or exceeds the permitted use, you will need to obtain permission directly from the copyright holder. To view a copy of this licence, visit http://creativecommons. org/licenses/by/4.0/.

\section{References}

1. Zhao J, Li L, Wang Y, Sutherland JW (2019) Impact of surface machining complexity on energy consumption and efficiency in CNC milling. Int J Adv Manuf Technol 102(9-12):2891. https://doi.org/10.1007/s00170-019-03334-9

2. Camposeco-Negrete C, de Dios Calderón-Nájera J (2019) Sustainable machining as a mean of reducing the environmental impacts related to the energy consumption of the machine tool: a case study of AISI 1045 steel machining. Int J Adv Manuf Technol 102(1-4):27. https://doi.org/10.1007/s00170-018-3178-0

3. Ma F, Zhang H, Cao H, Hon KKB (2017) An energy consumption optimization strategy for CNC milling. Int J Adv Manuf Technol 90(5-8):1715. https://doi.org/10.1007/s00170-016-9497-0

4. Kucukvar M, Cansev B, Egilmez G, Onat NC, Samadi H (2016) Energy-climate-manufacturing nexus: new insights from the regional and global supply chains of manufacturing industries. Appl Energy 184:889. https://doi.org/10.1016/j.apenergy.2016.03.068. https://www.sciencedirect.com/science/article/pii/S0306261916303889

5. European Commission, Energy Efficiency Plan 2011. Tech. rep. (2011). SEC(2011) 277 final

6. European Commission. 2030 climate \& energy framework (2019). https://ec.europa.eu/clima/policies/strategies/2030_en

7. Carabin G, Wehrle E, Vidoni R (2017) A review on energy-saving optimization methods for robotic and automatic systems. Robotics 6(4). https://doi.org/10.3390/robotics6040039

8. Hirzinger G, Sporer N, Albu-Schäffer A, Hähnle M, Krenn R, Pascucci A, Schedl M (2002) DLR's torque-controlled light weight robot III - are we reaching the technological limits now? In: Proceedings - IEEE International Conference on Robotics and Automation, vol 2. IEEE, pp 1710-1716. https://doi.org/10.1109/ robot.2002.1014788

9. Yin H, Huang S, He M, Li J (2016) An overall structure optimization for a light-weight robotic arm. In: Proceedings 
of the 2016 IEEE 11th Conference on Industrial Electronics and Applications, ICIEA 2016. IEEE, pp 1765-1770. https://doi.org/10.1109/ICIEA.2016.7603872

10. Yin H, Liu J, Yang F (2019) Hybrid structure design of lightweight robotic arms based on carbon fiber reinforced plastic and aluminum alloy. IEEE Access 7:64932. https://doi.org/10.1109/ACCESS.2019. 2915363. https://ieeexplore.ieee.org/document/8708269/

11. Kim N, Yun S, Shin D (2019) A bioinspired lightweight wrist for high-DoF robotic prosthetic arms. IEEE/ASME Trans Mechatron 24(6):2674. https://doi.org/10.1109/TMECH.2019.2941279. https://ieeexplore.ieee.org/document/8836522/

12. Albu-Schäffer A, Haddadin S, Ott C, Stemmer A, Wimböck T, Hirzinger G (2007) The DLR lightweight robot: design and control concepts for robots in human environments. Ind Robot 34(5):376. https://doi.org/10.1108/01439910710774386

13. Hagn U, Nickl M, Jörg S, Passig G, Bahls T, Nothhelfer A, Hacker F, Le-Tien L, Albu-Schäffer A, Konietschke R, Grebenstein M, Warpup R, Haslinger R, Frommberger M, Hirzinger G (2008) The DLR MIRO: a versatile lightweight robot for surgical applications. Ind Robot 35(4):324. https://doi.org/10.1108/01439910810876427

14. Kim YJ (2015) Design of low inertia manipulator with high stiffness and strength using tension amplifying mechanisms. In: IEEE International Conference on Intelligent Robots and Systems, vol 2015. IEEE, pp 5850-5856. https://doi.org/10.1109/IROS. 2015.7354208

15. Aziz MA, Zhanibek M, Elsayed AS, Abdulrazic MO, Yahya S, Almurib HA, Moghavvemi M (2016) Design and analysis of a proposed light weight three DOF planar industrial manipulator. In: IEEE industry application society 52nd annual meeting: IAS 2016. IEEE. https://doi.org/10.1109/IAS.2016.7731858

16. Matsuki H, Nagano K, Fujimoto Y (2019) Bilateral drive gear-a highly backdrivable reduction gearbox for robotic actuators. IEEE/ ASME Trans Mechatron 24(6):2661. https://doi.org/10.1109/TMECH. 2019.2946403. https://ieeexplore.ieee.org/document/ 8867893/

17. Kapoor R, Parveen CM (2013) Comparative study on various KERS Lecture notes in engineering and computer science 3 LNECS, 1969

18. Gale S, Eielsen AA, Gravdahl JT (2015) Modelling and simulation of a flywheel based energy storage system for an industrial manipulator. In: Proceedings of the IEEE International Conference on Industrial Technology, vol 2015. IEEE, pp 332337. https://doi.org/10.1109/ICIT.2015.7125120

19. Meike D, Senfelds A, Ribickis L (2013) Power converter for DC bus sharing to increase the energy efficiency in drive systems. In: IECON Proceedings (Industrial Electronics Conference). IEEE, pp 7199-7204. https://doi.org/10.1109/IECON.2013.6700329

20. Boscariol P, Carabin G, Gasparetto A, Lever N, Vidoni R (2015) Energy-efficient point-to-point trajectory generation for industrial robotic machines. In: Proceedings of the ECCOMAS Thematic Conference on Multibody Dynamics 2015, Multibody Dynamics 2015, pp 1425-1433

21. Richiedei D, Trevisani A (2016) Analytical computation of the energy-efficient optimal planning in rest-to-rest motion of constant inertia systems. Mechatronics 39:147. https://doi.org/10.1016/j.mechatronics.2016.05.004

22. Hansen C, Öltjen J, Meike D, Ortmaier T (2012) Enhanced approach for energy-efficient trajectory generation of industrial robots. In: IEEE International Conference on Automation Science and Engineering. IEEE, pp 1-7. https://doi.org/10.1109/CoASE. 2012.6386343

23. Wang X, Sun W, Li E, Song (2018) Energy-minimum optimization of the intelligent excavating process for large cable shovel through trajectory planning. Struct Multidiscip Optim 58(5):2219. https://doi.org/10.1007/s00158-018-2011-6
24. Boscariol P, Richiedei D (2019) Trajectory design for energy savings in redundant robotic cells. Robotics. 8(1). https://doi. org/10.3390/robotics8010015. https://www.scopus.com/inward/ record.uri?eid=2-s2.0-85063457172\&doi $=10.3390 \% 2$ Frobotics 80 10015\&partnerID $=40 \&$ md5=e26fffa08c7cbbc539d04724a740ea9b

25. Ayten KK, Sahinkaya MN, Dumlu A (2016) Optimum trajectory generation for redundant/hyper-redundant manipulators. IFACPapersOnLine 49(21):493. https://doi.org/10.1016/j.ifacol.2016. 10.651

26. Liu C, Cao GH, Qu YY, Cheng YM (2020) An improved PSO algorithm for time-optimal trajectory planning of Delta robot in intelligent packaging. Int J Adv Manuf Technol 107(3-4):1091. https://doi.org/10.1007/s00170-019-04421-7

27. Boscariol P, Richiedei D (2019) Energy-efficient design of multipoint trajectories for Cartesian robots. Int J Adv Manuf Technol 102(5-8):1853. https://doi.org/10.1007/s00170-018-03234-4

28. Jiang $X$, Lu W, Zhang Z (2018) An approach for improving the machining efficiency and quality of aerospace curved thin-walled parts during five-axis NC machining. Int J Adv Manuf Technol 97(5-8):2477. https://doi.org/10.1007/s00170-018-2129-0

29. Carabin G, Vidoni R, Wehrle E (2019) Energy saving in mechatronic systems through optimal point-to-point trajectory generation via standard primitives. In: Mechanisms and machine science, vol 68, pp 20-28. https://doi.org/10.1007/978-3-030-03320-0_3

30. Chen KY, Huang MS, Fung RF (2014) Dynamic modelling and input-energy comparison for the elevator system. Appl Math Model 38(7-8):2037. https://doi.org/10.1016/j.apm.2013.10.026

31. Khalaf P, Richter H (2019) Trajectory optimization of robots with regenerative drive systems: numerical and experimental results. IEEE Trans Robot. https://doi.org/10.1109/TRO.2019.2923920

32. Hansen C, Kotlarski J, Ortmaier T (2016) A concurrent optimization approach for energy efficient multiple axis positioning tasks. J Control Decis 3(4):223. https://doi.org/10.1080/23307706.2016. 1208548

33. Barreto JP, Corves B (2019) Resonant delta robot for pick-andplace operations. In: Mechanisms and machine science, vol 73, pp 2309-2318. https://doi.org/10.1007/978-3-030-20131-9_228

34. Carabin G, Palomba I, Wehrle E, Vidoni R (2020) Energy expenditure minimization for a delta-2 robot through a mixed approach. In: Computational methods in applied sciences, vol 53, pp 383-390. https://doi.org/10.1007/978-3-030-23132-3_46

35. Shushtari M, Nasiri R, Yazdanpanah MJ, Ahmadabadi MN (2017) Compliance and frequency optimization for energy efficiency in cyclic tasks. Robotica 35(12):1. https://doi.org/10.1017/ S0263574717000030

36. Palomba I, Wehrle E, Carabin G, Vidoni R (2020) Minimization of the energy consumption in industrial robots through regenerative drives and optimally designed compliant elements. Applied Sciences (Switzerland) 10(21):1-18. art. no 7475, https://doi.org/10. 3390/app10217475

37. Scalera L, Palomba I, Wehrle E, Gasparetto A, Vidoni R (2019) Natural motion for energy saving in robotic and mechatronic systems. Appl Sci (Switzerland) 9(17):3516. https://doi.org/10. 3390/app9173516

38. Li HZ, Gong ZM, Lin W, Lippa T Motion profile planning for reduced jerk and vibration residuals (2007). papers2://publication/uuid/FD6384DF-7479-45BC-A161-BB7169BBD6 AF

39. Duesterhoeft WC, Schulz MW, Clarke E (1951) Determination of instantaneous currents and voltages by means of alpha, beta, and zero components. Trans Amer Ins Elect Eng 70(2):1248. https://doi.org/10.1109/T-AIEE.1951.5060554. http://ieeexplore. ieee.org/document/5060554/

Publisher's note Springer Nature remains neutral with regard to jurisdictional claims in published maps and institutional affiliations. 